\title{
Artificial Heart Valve Testing Setup
}

\author{
Kamal K. Kar and Mridul Bharadwaj \\ IIT Kanpur \\ Email:*kamalkk@iitk.ac.in, \# mridul@iitk.ac.in
}

Artificial Heart Valves are prosthetics which are designed to replicate the function of our natural heart valves. Valvular heart diseases are now a major cause of mortality and morbidity also it is the second most common problem in cardiac surgery and third most common problem in cardiology.

The recent developments in the area of heart valve replacement surgery by Mechanical heart valves (MHV) have contributed towards the society very much. Being a crucial component, the Mechanical heart valve itself has to go through a rigorous testing procedure, which ensures the safe operation of the heart valve inside a human body. Testing of heart valve involves a serious challenge of replicating the inside heart conditions artificially in a lab for sustained time period and collecting useful data for comparison. Due to the high fatigue strength requirements of the heart valve, Accelerated testing (AT) of them is done. By doing accelerated testing sufficient data can be obtained within short time period to estimate valve's live within a human body.

Most of the current designs of a heart valve tester uses water as their working fluid and are operated using piston cylinder operated pumping actions (ex; Model MDS, Blockwise Eng.), some more sophisticated systems use pulse duplicator to mimic the inside heat flows (ex SD513 M6, Dynatek labs). But all these designs are good only when AT is considered, which a must to check for the fatigue strength is. None of the existing design can check for the actual flow conditions during a normal operating cycle actually happening inside a heart. This is crucial since slight changes in the flow due to artificial heart valve may have adverse effect on our body.

In view of this, the proposal is to make a optically transparent pulse operated cardiovascular simulator, which would enable us to measure, study and analyze various flow parameter associated with heart, like aortic flow, aortic pressure, mitral flow, mitral pressure and ventricular pressure etc. Optical nature of the setup would also enable us to do laser, ultrasound or photographic recording capabilities. The existing designs do not have their internal structure similar to a heart; this would be taken care of in the new design. So in addition to AT we would also be able to do the actual flow visualization in presence of the artificial valve. Also provisions would be made in the setup to change the working fluid and also provisions would be made to test for different pumping conditions like running, sleeping, etc. This would allow for a more detailed analysis of the valve.

(The device would be designed in accordance with the ISO 5840 and the US FDA regulations for the hemodynamic evaluation of prosthetic heart valves.)

\section{References}

1. Chapter - 13, On Accelerated Fatigue Testing of Prosthetic Heart Valves, Po-Chein Lu, Baoshu Xi, Shawyan Li, Jia Wu, Ned H. C. Hwang.

2. Dynatek Labs, SD 513 M6 heart valve tester, www.dynateklabs.com

3. Heart Valve Surgery - An Illustrated Guide, Jan Dominik and Pavel Zacek. 